\title{
Increased macrophage infiltration and neovascularization in congenital bicuspid aortic valve stenosis
}

\author{
Pedro R. Moreno, MD, ${ }^{\mathrm{a}}$ Luis Astudillo, MD, ${ }^{\mathrm{a}}$ Sammy Elmariah, MD, MPH, ${ }^{\mathrm{a}, \mathrm{b}}$ \\ K. Raman Purushothaman, MD, ${ }^{\mathrm{a}}$ Meeranani Purushothaman, $\mathrm{PhD},{ }^{\mathrm{a}}$ Patrick A. Lento, MD, ${ }^{\mathrm{c}}$ \\ Samin K. Sharma, MD, ${ }^{a}$ Valentin Fuster, MD, PhD, ${ }^{\mathrm{a}, \mathrm{d}}$ and David H. Adams, $\mathrm{MD}^{\mathrm{e}}$
}

\begin{abstract}
Objectives: Patients with congenital bicuspid aortic valves have aortic valve stenosis at a relatively young age compared with patients with tricuspid aortic valves. We hypothesize that aortic valve stenosis evolves from a more aggressive inflammatory process, with increased macrophage/T-cell and neovessel content in congenital bicuspid aortic valveswhen compared with that seen in tricuspid valves.
\end{abstract}

\begin{abstract}
Methods: Fifty-one severely stenotic aortic valves were obtained at the time of aortic valve replacement. A total of 17 bicuspid and 34 tricuspid aortic valves were evaluated. Macrophage/T-cell infiltration (CD68 plus CD3) and neovessel density (CD34) were evaluated with immunohistochemical staining. Leaflet calcification and ossification were also quantified. Real-time polymerase chain reaction was used to assess expression of chondromodulin 1 and vascular endothelial growth factor.
\end{abstract}

\begin{abstract}
Results: The density of macrophages/T cells was greater in congenital bicuspid aortic valves than in tricuspid valves (51 \pm 31 vs $23 \pm 13$ cells $/ \mathrm{mm}^{2}, P=.002$ ). Neovascularization was more frequently noted in congenital bicuspid aortic valves when compared with tricuspid valves ( $31 \pm 10$ vs $21 \pm 9$ vessels $\left./ \mathrm{mm}^{2}, P=.0005\right)$, and calcification was more severe $(P=.03)$. Expression of chondromodulin 1 demonstrated a 6 -fold downregulation $(P=.0003)$ and expression of vascular endothelial growth factor demonstrated a 2-fold increase $(P=.02)$ in congenital bicuspid aortic valves compared with that seen in tricuspid valves. Multivariable analyses demonstrated significant associations between bicuspid aortic valve anatomy and increased inflammatory cell infiltration $(\beta=25.8, P=.0007)$ and neovascularization $(\beta=9.4, P=.001)$, despite adjusting for measured covariates.

Conclusions: The pathogenesis of aortic valve stenosis in bicuspid aortic valves is associated with a more aggressive inflammatory process with increased macrophage infiltration and neovascularization when compared with that seen in tricuspid valves. (J Thorac Cardiovasc Surg 2011;142:895-901)
\end{abstract}

A congenital bicuspid aortic valve (CBAV) is one of the most common congenital cardiovascular malformations, occurring in as much as $2 \%$ of adults, ${ }^{1}$ and a large proportion of patients with CBAVs will require valvular intervention for aortic valve stenosis (AS), regurgitation, or infective endocarditis. ${ }^{2}$ AS is the most frequent complication of CBAVs, frequently necessitating aortic valve replacement surgery relatively early in life when compared with patients with tricuspid aortic valves (TAVs). The early

\footnotetext{
From the Zena and Michael A. Weiner Cardiovascular Institute ${ }^{\mathrm{a}}$ and the Departments of Pathology ${ }^{\mathrm{c}}$ and Cardiothoracic Surgery, ${ }^{\mathrm{e}}$ Mount Sinai School of Medicine, New York, NY; the Cardiology Division, ${ }^{\mathrm{b}}$ Massachusetts General Hospital, Harvard Medical School, Boston, Mass; and Centro Nacional de Investigaciones Cardiovasculares (CNIC), ${ }^{\mathrm{d}}$ Madrid, Spain.

Disclosures: Dr. Elmariah was supported by the Glorney-Raisbeck Fellowship Program, the Corlette Glorney Foundation, and the New York Academy of Medicine; by the GlaxoSmithKline Research and Education Foundation for Cardiovascular Disease; and by a grant from the National Heart, Lung, and Blood Institute (T32 HL007824).

Received for publication Sept 13, 2010; revisions received Dec 23, 2010; accepted for publication March 1, 2011; available ahead of print April 11, 2011.

Address for reprints: Pedro R. Moreno, MD, Mount Sinai School of Medicine, Zena and Michael A. Weiner Cardiovascular Institute, One Gustave L. Levy Place, Box 1030, New York, NY 10029 (E-mail: pedro.moreno@msnyuhealth.org). $0022-5223 / \$ 36.00$

Copyright (c) 2011 by The American Association for Thoracic Surgery doi:10.1016/j.jtcvs.2011.03.002
}

onset of symptomatic AS reflects the more rapidly progressing valve stenosis frequently seen in those with CBAVs. ${ }^{3}$

To date, few data exist to explain the early development of AS in patients with CBAVs. The progression of AS in patients with CBAVs differs based on the symmetry and anatomy of the bicuspid valve, suggesting that stress-strain relations might be responsible for early disease onset and more rapid progression. ${ }^{3,4}$ Cardiovascular risk factors, such as hypercholesterolemia and a history of hypertension, have been associated with increased risk of AS in those with CBAVs. ${ }^{5}$ Significant data are mounting that suggest that the genetic aberrations that lead to bicuspid anatomy might also lead to an increased propensity to calcify. ${ }^{6-10}$ Such data suggest similarities between the mechanisms responsible for AS in bicuspid and tricuspid aortic valves. Fibrocalcific AS in TAVs results from an active process that, much like atherosclerosis, is preceded by basement membrane disruption, inflammatory cell infiltration, neovascularization, and lipid deposition and culminates in severe calcification and bone formation. ${ }^{1-15}$ Together, these data suggest that the hemodynamic and cellular abnormalities associated with CBAVs might intensify the active processes involved in valve degeneration. Here we hope to determine whether inflammation, defined by 


$$
\begin{aligned}
& \text { Abbreviations and Acronyms } \\
& \begin{aligned}
\text { AS } & =\text { aortic valve stenosis } \\
\text { BMP } & =\text { bone morphogenic protein } \\
\text { CBAV } & =\text { congenital bicuspid aortic valve } \\
\text { ChM-1 } & =\text { chondromodulin } 1 \\
\text { CI } & =\text { confidence interval } \\
\text { NOTCH1 } & =\text { NOTCH homologue- } 1 \\
\text { TAV } & =\text { tricuspid aortic valve } \\
\text { VEGF } & =\text { vascular endothelial growth factor }
\end{aligned}
\end{aligned}
$$

inflammatory cell infiltration and neovascularization and concomitant calcification, is increased in patients with AS caused by CBAVs. Additionally, we assess the expression of chondromodulin 1 (ChM-1), a potent antiangiogenic factor, and vascular endothelial growth factor (VEGF), a stimulator of angiogenesis, in stenotic bicuspid aortic valves and tricuspid aortic valves.

\section{MATERIALS AND METHODS \\ Study Population and Data Collection}

From January to December 2006, 92 patients with severe aortic stenosis underwent successful aortic valve replacement at the Mount Sinai Medical Center. All bicuspid valves $(n=20)$ and randomly selected tricuspid valves $(\mathrm{n}=34)$ were selected for further analysis. Valve tissue was collected at the time of the operation. Patients' medical records were reviewed to assess the prevalence of cardiovascular risk factors and the use of relevant cardiovascular medications. The retrospective review of clinical data was approved by the Mount Sinai School of Medicine's Institutional Review Board.

\section{Immunohistochemistry}

Four-micrometer serial sections of formalin-fixed, paraffin-embedded valve tissue were cut for subsequent staining. Tissue sections were deparaffinized and pretreated with sodium citrate antigen retrieval for 5-minute intervals up to a total of 15 minutes. After blocking endogenous peroxidase activity with $10 \%$ hydrogen peroxide in methanol, sections were subjected to double labeling. Primary monoclonal murine anti-human antibodies of IgG1 class (DAKO Corp, Carpinteria, Calif) included the CD34 endothelial clone Qbend-10 at 1:30 dilution, the CD68 macrophage clone KP-1 at 1:100 dilution/CD3 T lymphocyte clone at 1:50 dilution, and the $\alpha$-actin smooth muscle clone at 1:50 dilution. Affinity-purified secondary anti-mouse IgG antibodies with Western blotting-confirmed specificity were obtained from Vector Laboratories (Burlingame, Calif) as the Avidin-Biotin Conjugated Elite kit PK6102. Double immunohistochemistry was systematically performed in all sections by applying 2 entirely separate sets of contrasting bicolor preparations to each block. The first paired bicolor preparation used blue (alkaline phosphatase SK 5300 Vector) and red (alkaline phosphatase SK 5100 Vector) chromogen reaction to distinguish endothelial microvessel cells from inflammatory cells (macrophage-lymphocyte cocktail), respectively. Specificity of all antibodies was confirmed by means of routine positive and negative controls for each stain by using human tonsil and bowel tissue to exclude nonspecific binding of the primary antibody.

Macrophages and $\mathrm{T}$ lymphocytes were defined as $\mathrm{CD}^{+} 8^{+} / \mathrm{CD} 3^{+}$mononuclear round cells identified by means of light microscopy, as previously described. ${ }^{16}$ Neovessels were defined as tubuloluminal CD $34^{+}$capillaries recognized in cross-sectional and longitudinal profiles, as previously described. ${ }^{17}$ The aortic valve specimen was systematically examined in 10 random microscopic fields and sequentially planimetered by means of dedicated image-processing software (Quantim; Zedec Technologies, Burlington, NC) at $40 \times$ magnification, as previously described. ${ }^{18}$ The density of inflammatory cell infiltration and of neovessels was determined by dividing the number of inflammatory cells and neovessels per field by the individual area examined. Mean density was calculated for each specimen and used for subsequent analyses. Calcification was graded on a scale of 1 to 4 , as previously described. ${ }^{19}$ Briefly, grade 1 valves possessed no calcification, grade 2 valves possessed early calcific nodules, grade 3 valves possessed several calcific nodules with mild structural distortion, and grade 4 valves possessed many calcific nodules with severe structural distortion. Ossification was identified by means of osteoid matrix deposition or lamellar bone formation ${ }^{12}$ and was analyzed as a dichotomous variable. All measurements were performed blind to valve morphology.

\section{Gene Expression}

An Arcturus Paradise Plus Whole Transcript RT kit (MDS Analytical Technologies, Sunnyvale, Calif) was used to isolate RNA from formalinfixed paraffin-embedded tissue samples, as we have previously described. ${ }^{20}$ Briefly, the tissue sections were deparaffinized with xylene and then digested with proteinase $\mathrm{K}$ for 16 to 20 hours at $37^{\circ} \mathrm{C}$ in an oven. Total RNA was then isolated with the Paradise reagent system (MDS Analytical Technologies). The quality of the isolated RNA was verified with the Paradise sample quality assessment kit (MDS Analytical Technologies), and the concentration of RNA was quantified with Nanodrop (Thermo Fisher Scientific, Waltham, Mass). The cDNA was prepared by using reverse transcriptase polymerase chain reaction. Gene expression of ChM-1 and VEGF was assessed by using quantitative real-time polymerase chain reaction with an ABI PRISM 7700 Sequence Detection System (Applied Biosystems, Foster City, Calif). Specific primer pairs for ChM-1 used were as follows: forward $5^{\prime}$-tcagcetgtgaaggacaaca- $3^{\prime}$ and reverse $5^{\prime}$-taggaaggt caccgcagagt- $3^{\prime}$ and for VEGF were forward $5^{\prime}$-ctacctccaccatgccaagt- $3^{\prime}$ and reverse $5^{\prime}$-atgattctgcctcctcctt-3. The mRNA expression of these genes was normalized to that of the housekeeping gene $\beta$-actin.

\section{Statistical Analysis}

All data are presented as means \pm standard deviations. For 2-group comparisons, Gaussian samples were compared by using the 2-tailed Student $t$ test preceded by Levene's $\mathrm{F}$ test for equality of variance. NonGaussian samples were compared with the Mann-Whitney nonparametric test. Fisher's exact test was used to test the significance of association for discrete variables. Multivariable linear regression modeling was used to adjust for suspected confounders. Given our small sample size, we limited multivariable models to 6 covariates. The most relevant covariates were selected for inclusion in multivariable models based on a priori clinical knowledge. Statistical analyses were performed with SAS software (version 9.1.3; SAS Institute, Inc, Cary, NC). Two-sided $P$ values of less than .05 were considered statistically significant.

\section{RESULTS \\ Patients' Characteristics}

A total of 51 patients were included in this analysis (Table 1). Three bicuspid valves were excluded because of either rheumatic valve disease or infectious endocarditis. The study population included $31(61 \%)$ men. The overall mean age was $68 \pm 10$ years. Nineteen $(37 \%)$ of the patients were diabetic, $45(88 \%)$ had hypertension, and $38(75 \%)$ had hypercholesterolemia. Coronary artery disease was present in $33(65 \%)$, with $22(57 \%)$ undergoing coronary artery bypass surgery. Chronic kidney disease was present in 9 $(18 \%)$ patients. Hydroxymethyl-glutaryl coenzyme A reductase inhibitors (statins) were used in 29 (57\%) patients, 
TABLE 1. Clinical and demographic characteristics of patients with bicuspid and tricuspid aortic stenosis

\begin{tabular}{|c|c|c|c|c|}
\hline & All patients, $n(\%[n=51])$ & Bicuspid, $\mathbf{n}(\%[n=17])$ & Tricuspid, $\mathbf{n}(\%[\mathrm{n}=34])$ & $P$ value \\
\hline Age (y) & $68 \pm 10$ & $56 \pm 8$ & $67 \pm 10$ & .0004 \\
\hline Female sex & $20(39)$ & $3(18)$ & $17(50)$ & .03 \\
\hline \multicolumn{5}{|l|}{ Risk factors } \\
\hline Hypertension & $45(88)$ & $15(88)$ & $30(88)$ & 1 \\
\hline Hypercholesterolemia & $38(75)$ & $13(76)$ & $25(78)$ & 1 \\
\hline Diabetes mellitus & $19(37)$ & $6(35)$ & $13(38)$ & 1 \\
\hline Chronic kidney disease & $9(18)$ & $2(12)$ & $7(21)$ & .70 \\
\hline Tobacco use & $24(47)$ & $12(70)$ & $12(35)$ & .04 \\
\hline \multicolumn{5}{|l|}{ Coronary artery disease } \\
\hline Angiography & $33(65)$ & $10(59)$ & $23(68)$ & .55 \\
\hline Coronary bypass surgery & $29(57)$ & $9(52)$ & $20(59)$ & .77 \\
\hline \multicolumn{5}{|l|}{ Medical therapy } \\
\hline Statin & $29(57)$ & $10(59)$ & $19(56)$ & 1 \\
\hline ACEI & $24(47)$ & $8(47)$ & $16(47)$ & 1 \\
\hline ARB & $9(18)$ & $2(12)$ & $7(21)$ & .70 \\
\hline \multicolumn{5}{|l|}{ Echocardiographic parameters } \\
\hline $\operatorname{LVEF}(\%)$ & $51 \pm 12$ & $53 \pm 11$ & $51 \pm 13$ & .56 \\
\hline $\operatorname{AVA}\left(\mathrm{cm}^{2}\right)$ & $0.78 \pm 0.2$ & $0.84 \pm 0.3$ & $0.75 \pm 0.2$ & .31 \\
\hline Mean gradient $(\mathrm{mm} \mathrm{Hg})$ & $47 \pm 20$ & $46 \pm 21$ & $47 \pm 20$ & .88 \\
\hline Aortic root diameter $(\mathrm{cm})$ & $3.1 \pm 0.4$ & $3.2 \pm 0.5$ & $3.0 \pm 0.4$ & .22 \\
\hline
\end{tabular}

$A C E I$, Angiotensin-converting enzyme inhibitor; $A R B$, angiotensin receptor blocker; LVEF, left ventricular ejection fraction; $A V A$, aortic valve area.

angiotensin-converting enzyme inhibitors were used in 24 $(47 \%)$ patients, and angiotensin II receptor blockers were used in $9(18 \%)$ patients.

When stratified by valve morphology, patients with bicuspid and tricuspid aortic valves were comparable, with a few notable exceptions. As expected, patients with CBAVs were younger than patients with a tricuspid valves (61 \pm 8 vs $71 \pm 10$ years, $P=.0004)$. In concert with prior epidemiologic data, ${ }^{2}$ patients with CBAVs were more often men $(82 \%$ vs $50 \%, P=.03)$. Additionally, a significantly greater prevalence of tobacco use was seen in patients with CBAVs compared with that seen in those with TAVs (70\% vs $35 \%$, respectively; $P=.04$ ).

\section{Echocardiographic Measurements}

In all included patients the mean left ventricular ejection fraction was $51 \% \pm 12 \%$. The mean aortic valve area was $0.78 \pm 0.23 \mathrm{~cm}^{2}$, and the mean transvalvular gradient was $47 \pm 20 \mathrm{~mm} \mathrm{Hg}$. When comparing patients with CBAVs and those with TAVs, no significant differences in echocardiographic measures of aortic stenosis severity were observed.

\section{Histopathologic Assessment}

The severity of inflammatory cell infiltration and neovascularization differed significantly between patients with CBAVs and those with TAVs (Figure 1). The density of macrophages/T cells was greater in patients with CBAVs compared with that seen in those with TAVs $(51 \pm 31$ vs $23 \pm 13$ cells $/ \mathrm{mm}^{2} ; P=.002$; Figure $2, A$ ). Neovasculariza- tion was also more frequently noted in patients with CBAVs when compared with those with TAVs $(31 \pm 10$ vs $21 \pm 9$ vessels $/ \mathrm{mm}^{2} ; P=.0005$; Figure $\left.2, B\right)$. Valve calcification was present in all valves, with more severe calcification noted in CBAVs $(P=.03$, Table 2$)$. The proportion of valves containing ossification was greater in CBAVs, but this difference was not statistically significant $(35 \%$ vs $21 \%, P=.31)$.

Valve inflammation, as defined by the density of inflammatory cell infiltration, demonstrated a positive correlation with density of neovascularization and extent of valve calcification in both tricuspid $(r=0.49$ and $P=.003$ vs $r=0.57$ and $P=.0004$, respectively) and bicuspid ( $r=0.58$ and $P=.02$ vs $r=0.53$ and $P=.04$, respectively) valves. Ossified bicuspid valves demonstrated increased inflammatory cell infiltration $\left(72 \pm 29\right.$ vs $40 \pm 26$ cells $/ \mathrm{mm}^{2}$, $P=.04)$, but this relationship was not seen in tricuspid valves $\left(30 \pm 10\right.$ vs $22 \pm 14$ cells $\left./ \mathrm{mm}^{2}, P=.13\right)$. No significant relationship was observed between neovascularization and either extent of calcification or ossification.

Multivariable analyses were performed to minimize the effects of potential confounders. Despite adjusting for age, sex, presence of chronic kidney disease, smoking status, and use of statins, bicuspid anatomy was strongly associated with increased aortic valve inflammatory cell infiltration $(\beta=25.8[95 \%$ confidence interval $(\mathrm{CI})$, 11.5-40.0], $P=.0007)$ and neovascularization $(\beta=9.4$ [95\% CI, 3.9-14.8], $P=.001$; Table 3). Significant associations were also noted between neovascularization and the presence of chronic kidney disease and male sex (Table 3). 


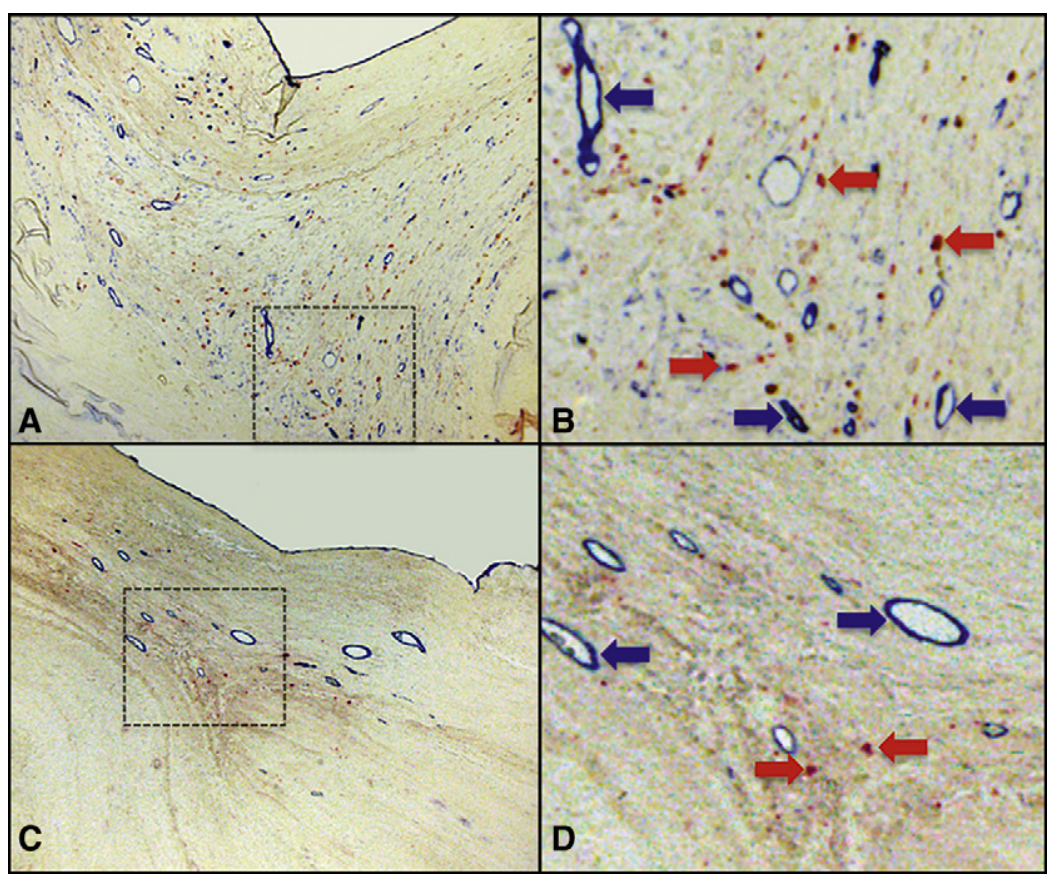

FIGURE 1. Immunohistochemical staining of severely stenotic bicuspid and tricuspid aortic valves. Macrophages and T lymphocytes were stained with anti-CD68/CD3 monoclonal antibodies conjugated to a red chromogen (red arrows). Neovessels were selectively stained with a monoclonal antibody to the endothelial cell marker CD34 conjugated with a blue chromogen (blue arrows). More inflammatory cell infiltration and neovascularization is noted in bicuspid (A and B) compared with tricuspid (C and D) valves. The aortic side of the aortic valve is depicted.

Because of the significant effect of chronic kidney disease on both valve inflammation and neovascularization, we conducted an ad hoc multivariable analysis excluding valve specimens from patients with chronic kidney disease. Despite this exclusion, we found that the association of bicuspid aortic valve anatomy with inflammatory cell infiltration and neovascularization remained relatively unchanged ( $\beta=25.0$ [95\% CI, 8.9-41.0] and $P=.003$ vs $\beta=11.7$ [95\% CI, 6.1-17.3] and $P=.0002$, respectively).

\section{Gene Expression}

Because of heavy calcification and formalin fixation, we were able to extract RNA from only 12 of 51 aortic valves (6 TAVs and 6 CBAVs). ChM-1 gene expression was significantly downregulated by more than 6-fold in CBAVs compared with that seen in TAVs $(P=.0003$; Figure $2, C)$. Conversely, VEGF gene expression in bicuspid valves was approximately twice that seen in tricuspid valves $(P=.02$; Figure $2, D)$.

\section{DISCUSSION}

This study identified a $122 \%$ increase in inflammatory cell density, a $48 \%$ increase in neovascularization, and more severe calcification in bicuspid valves compared with tricuspid valves. Bicuspid anatomy was associated with inflammatory cell infiltration and neovascularization, despite adjusting for age, sex, chronic kidney disease, smoking status, and use of statins. Additionally, ChM-1 expression was significantly downregulated and VEGF expression was significantly upregulated in CBAVs, findings in agreement with the discrepant neovascularization seen on histopathological assessments. These findings suggest that remodeling of CBAVs is associated with a more aggressive inflammatory process. Although our study does not allow inference of causality, it does provide useful insight into the mechanisms at play within the stenotic bicuspid aortic valve.

Fibrocalcific AS is now known to be the consequence of an active process similar to that responsible for atherosclerosis. Both are initiated by basement membrane disruption and lipid deposition and are mediated by inflammatory cell infiltration and neovascularization. ${ }^{11-13}$ Moreover, many of the typical risk factors for atherosclerosis have also been associated with AS. ${ }^{11}$ Accelerated progression of AS in patients with CBAVs has also been associated with atherosclerotic risk factors, suggesting that a similar active process is involved. ${ }^{5}$

In patients with atherosclerosis, shear stress plays a role in endothelial disruption and in plaque size and composition through modulation of inflammatory chemokines. ${ }^{21,22}$ Stress-strain relations might similarly play a role in the accelerated development of AS in patients with CBAVs given the relationship between rate of progression and valve symmetry and anatomy. ${ }^{3,4}$ These data suggest that increased shear stress in bicuspid valves might modulate inflammatory processes within the valve, as it does in 

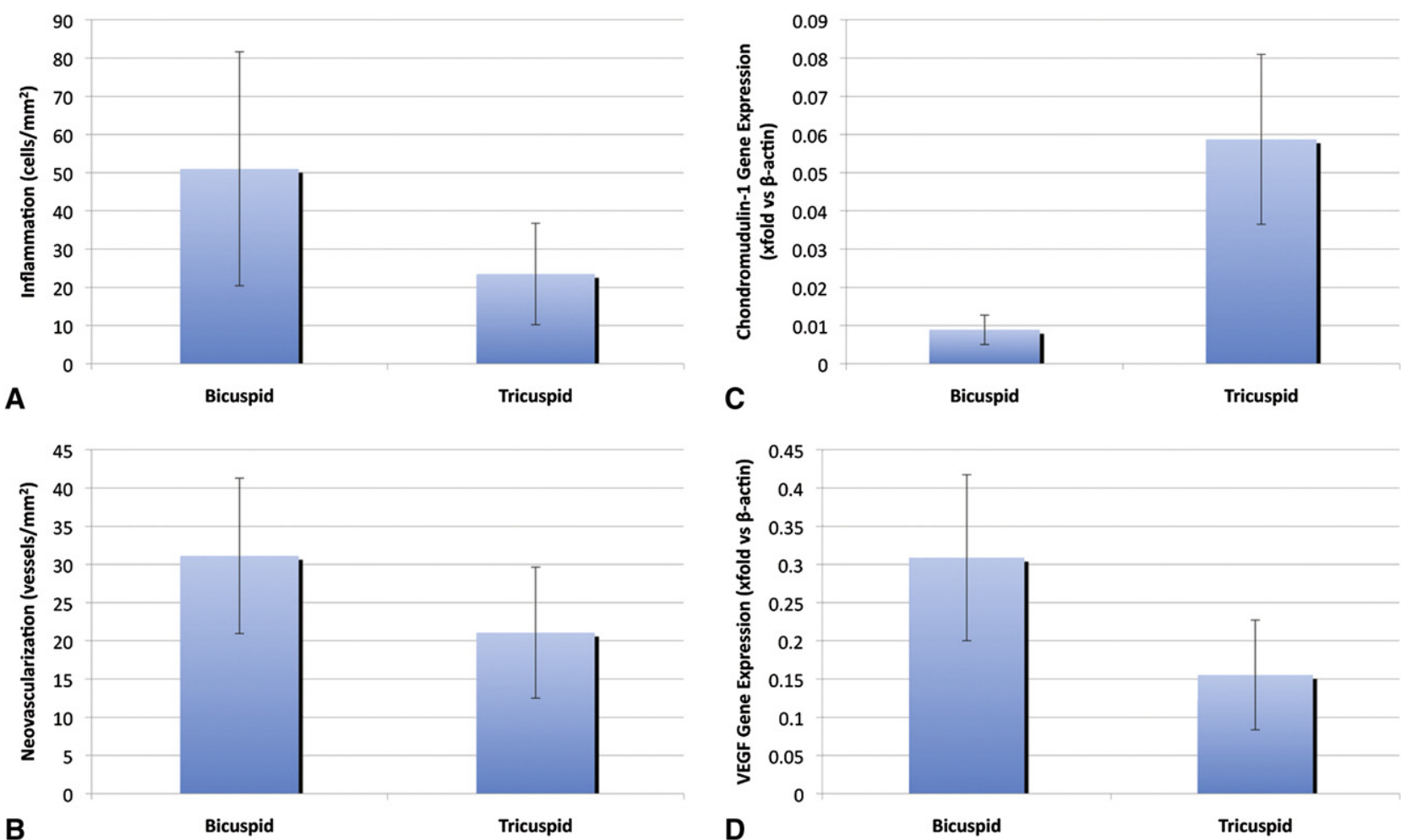

FIGURE 2. Inflammation and neovascularization in bicuspid and tricuspid aortic valve specimens. A, The mean density of inflammatory cells (cells per square millimeter) noted in bicuspid aortic valves was significantly greater than that seen in tricuspid valves $(P=.002)$. B, The mean density of neovessels (vessels per square millimeter) was also significantly greater in bicuspid aortic valves compared with that seen in tricuspid valves $(P=.0005)$. Error bars denote standard deviations. C, Gene expression of chondromodulin 1, a potent antiangiogenic factor, is significantly downregulated in congenital bicuspid aortic valves compared with that seen in tricuspid aortic valves $(P=.0003)$. D, However, expression of vascular endothelial growth factor $(V E F G)$, which is proangiogenic, was upregulated in congenital bicuspid aortic valves $(P=.02)$.

atherosclerosis. In fact, recent data from Sucosky and colleagues $^{23}$ suggest the presence of a shear stress-induced inflammatory pathway. Nonphysiologic pulsatile shear stress stimulated endothelial expression of vascular cell adhesion molecule 1, intracellular adhesion molecule 1, bone morphogenic protein (BMP) 4, and transforming growth factor $\beta 1$ on the aortic sides of tricuspid porcine valves. Moreover, Mohler and associates ${ }^{12}$ demonstrated increased BMP-2 and BMP-4 expression and ossification in areas adjacent to neovascularization and inflammatory cell infiltration.

Alternate hypotheses have been proposed to explain the accelerated calcification seen in CBAVs. Mutations in the

TABLE 2. Severity of aortic valve calcification is greater in bicuspid aortic valves

\begin{tabular}{lccc}
\hline $\begin{array}{c}\text { Severity of } \\
\text { calcification }\end{array}$ & $\begin{array}{c}\text { Bicuspid } \\
(\mathbf{n}=\mathbf{1 7})\end{array}$ & $\begin{array}{c}\text { Tricuspid } \\
(\mathbf{n = 3 4 )}\end{array}$ & $\boldsymbol{P}$ value \\
\hline Grade 1 & 1 & 0 & .03 \\
Grade 2 & 1 & 13 & \\
Grade 3 & 5 & 7 & \\
Grade 4 & 10 & 14 & \\
\hline
\end{tabular}

NOTCH homologue-1 (NOTCH1) gene have been associated with a spectrum of aortic valve disease, including CBAVs and heavy calcification of TAVs. ${ }^{6,10}$ NOTCH1 represses calcification pathways mediated by Runx2, a transcriptional regulator of osteoblastic differentiation, and BMP-2. Therefore mutations of NOTCH1 lead to deregulation of these calcification pathways. ${ }^{6}$ Additionally, multiscale computerized modeling of tricuspid and bicuspid aortic valves demonstrates that bicuspid valves undergo greater flexure and stronger jet formation than tricuspid valves; however, these forces do not transmit to the cellular and molecular levels. ${ }^{8}$ As such, it is hypothesized that the increased structural forces exerted on CBAVs are not capable of inducing calcific remodeling of the valve and that increased calcification might be due to genetic differences in matrix components. ${ }^{8}$ These recent findings suggest that accelerated calcification in CBAVs might be secondary to the genetic mutation causing the bicuspid morphology.

Whether shear stress, genetic factors, or both are responsible for the observed propensity of CBAVs to calcify, we found that inflammation and neovascularization are more severe in stenotic bicuspid valves than in tricuspid valves. Additionally, we are the first to evaluate ChM-1 expression 
TABLE 3. Multivariate analyses of aortic valve inflammation and neovascularization

\begin{tabular}{lccc}
\hline \multicolumn{1}{c}{ Parameter } & $\beta$-Coefficient & Standard error & $\boldsymbol{P}$ value \\
\hline Inflammation & & & \\
$\quad$ Bicuspid anatomy & 25.8 & 7.1 & .0007 \\
Age & -0.1 & 0.3 & .63 \\
Male sex & 10.2 & 6.4 & .12 \\
Chronic kidney disease & 12.6 & 8.5 & .15 \\
Statin use & 4.3 & 5.9 & .48 \\
Tobacco use & -1.2 & 6.6 & .86 \\
Neovascularization & & & \\
Bicuspid anatomy & 9.4 & 2.7 & .001 \\
Age & 0.03 & 0.1 & .80 \\
Male sex & 5.4 & 2.5 & .03 \\
Chronic kidney disease & 14.1 & 3.3 & $<.0001$ \\
Statin use & -0.9 & 2.3 & .70 \\
Tobacco use & 0.3 & 2.5 & .92 \\
\hline
\end{tabular}

in bicuspid valves. ChM-1 is a potent antiangiogenic factor that was recently found to play a vital role in maintaining normal valve function by inhibiting angiogenesis that can lead to dystrophic valve remodeling. ${ }^{24}$ Aortic valves from $\mathrm{ChM}-1^{-1-}$ mice demonstrated increased neovascularization, VEGF expression, lipid deposition, inflammation, and calcification, suggesting that neovascularization is the first step in valve degeneration. ${ }^{24}$ Similar changes have been observed in diseased human valves. ${ }^{1-13,24}$ Hence the significant reduction in ChM-1 expression in CBAVs, as noted in our small exploratory analysis, might be responsible for the observed increased in neovascularization in bicuspid valves.

Significant data support an association between neovascularization and inflammatory changes within the aortic valve with the calcification process. ${ }^{13,25}$ Monocytes and macrophages within valves secrete a variety of matrix proteins that activate valve myofibroblasts and trigger their osteoblastic transdifferentiation. ${ }^{11}$ This in part explains the proximity of inflammatory cells and neovessels to calcification foci within the valve and supports the notion that valve inflammation is responsible for subsequent calcification and stenosis. ${ }^{12,25}$ Here we extend these findings by demonstrating that stenotic bicuspid valves are associated with increased inflammatory cell infiltration and neovascularization when compared with tricuspid valves. We also demonstrate a positive correlation between valve inflammation, neovascularization, and the extent of valve calcification. Although additional data are needed to establish causality, our findings might help explain the greater prevalence and accelerated progression of AS and the increased valve calcification observed in patients with $\mathrm{CBAVs}^{3,9}$ and might in turn identify angiogenesis as a potential therapeutic target in patients with CBAVs.

This study reflects histopathological features of the final stages of calcific AS. As such, our study comments on asso- ciations among neovascularization, inflammation, and calcification but cannot definitively distinguish between cause and effect. Although these findings are useful and help further our understanding of valve degeneration, they are to be considered hypothesis generating. More definitive data on the progression of AS would necessitate histopathological examination of bicuspid and tricuspid aortic valves at varying stages of disease. Additionally, our study is limited in that a relatively small number of valves were studied. As such, we did not possess sufficient statistical power to adjust for all potential confounders. Larger studies are needed to confirm our findings and to further characterize differences between bicuspid and tricuspid aortic valve disease.

Severe aortic stenosis in bicuspid aortic valves is associated with a more aggressive inflammatory process with increased inflammatory cell infiltration and neovascularization when compared with tricuspid AS. These findings might help explain the more frequent onset and rapid progression of AS and the heavy aortic valve calcification seen in patients with CBAVs, although further study is needed to establish causation. Whether this aggressive inflammatory process is more amenable to antiangiogenic pharmacologic interventions remains to be elucidated.

We thank Dr Carlos Alviar for his assistance with data collection.

\section{References}

1. Ward C. Clinical significance of the bicuspid aortic valve. Heart. 2000;83:81-5

2. Michelena HI, Desjardins VA, Avierinos JF, Russo A, Nkomo VT, Sundt TM, et al. Natural history of asymptomatic patients with normally functioning or minimally dysfunctional bicuspid aortic valve in the community. Circulation. 2008; 117:2776-84.

3. Beppu S, Suzuki S, Matsuda H, Ohmori F, Nagata S, Miyatake K. Rapidity of progression of aortic stenosis in patients with congenital bicuspid aortic valves. Am J Cardiol. 1993;71:322-7.

4. Otto CM. Calcification of bicuspid aortic valves. Heart. 2002;88:321-2.

5. Chan KL, Ghani M, Woodend K, Burwash IG. Case-controlled study to assess risk factors for aortic stenosis in congenitally bicuspid aortic valve. Am J Cardiol. 2001;88:690-3.

6. Garg V, Muth AN, Ransom JF, Schluterman MK, Barnes R, King IN, et al. Mutations in NOTCH1 cause aortic valve disease. Nature. 2005;437:270-4

7. Nigam V, Srivastava D. Notch1 represses osteogenic pathways in aortic valve cells. J Mol Cell Cardiol. 2009; 47:828-34.

8. Weinberg EJ, Kaazempur Mofrad MR. A multiscale computational comparison of the bicuspid and tricuspid aortic valves in relation to calcific aortic stenosis. J Biomech. 2008;41:3482-7.

9. Isner JM, Chokshi SK, DeFranco A, Braimen J, Slovenkai GA. Contrasting histoarchitecture of calcified leaflets from stenotic bicuspid versus stenotic tricuspid aortic valves. J Am Coll Cardiol. 1990;15:1104-8.

10. McKellar SH, Tester DJ, Yagubyan M, Majumdar R, Ackerman MJ, Sundt TM 3rd. Novel NOTCH1 mutations in patients with bicuspid aortic valve disease and thoracic aortic aneurysms. J Thorac Cardiovasc Surg. 2007;134: 290-6.

11. Goldbarg SH, Elmariah S, Miller MA, Fuster V. Insights into degenerative aortic valve disease. J Am Coll Cardiol. 2007;50:1205-13.

12. Mohler ER III, Gannon F, Reynolds C, Zimmerman R, Keane MG, Kaplan FS. Bone formation and inflammation in cardiac valves. Circulation. 2001;103: 1522-8.

13. Soini Y, Salo T, Satta J. Angiogenesis is involved in the pathogenesis of nonrheumatic aortic valve stenosis. Hum Pathol. 2003;34:756-63.

14. Yu PJ, Skolnick A, Ferrari G, Heretis K, Mignatti P, Pintucci G, et al. Correlation between plasma osteopontin levels and aortic valve calcification: potential 
insights into the pathogenesis of aortic valve calcification and stenosis. $J$ Thorac Cardiovasc Surg. 2009;138:196-9.

15. Yang X, Meng X, Su X, Mauchley DC, Ao L, Cleveland JC Jr, et al. Bone morphogenic protein 2 induces Runx 2 and osteopontin expression in human aortic valve interstitial cells: role of Smad1 and extracellular signal-regulated kinase 1/2. J Thorac Cardiovasc Surg. 2009;138:1008-15.

16. Moreno PR, Bernardi VH, Lopez-Cuellar J, Murcia AM, Palacios IF, Gold HK, et al. Macrophages, smooth muscle cells, and tissue factor in unstable angina. Implications for cell-mediated thrombogenicity in acute coronary syndromes. Circulation. 1996;94:3090-7.

17. Moreno PR, Purushothaman KR, Fuster V, Echeverri D, Truszczynska H, Sharma SK, et al. Plaque neovascularization is increased in ruptured atherosclerotic lesions of human aorta. Implications for plaque vulnerability. Circulation. 2004;110:2032-8.

18. Bauters C, Asahara T, Zheng LP, Takeshita S, Bunting S, Ferrara N, et al. Site-specific therapeutic angiogenesis after systemic administration of vascular endothelial growth factor. J Vasc Surg. 1995;21:314-25.

19. Warren BA, Yong JL. Calcification of the aortic valve: its progression and grading. Pathology. 1997;29:360-8.
20. Levy AP, Purushothaman KR, Levy NS, Purushothaman M, Strauss M, Asleh R et al. Downregulation of the hemoglobin scavenger receptor in individuals with diabetes and the Hp 2-2 genotype: implications for the response to intraplaque hemorrhage and plaque vulnerability. Circ Res. 2007;101:106-10.

21. Cheng C, Tempel D, van Haperen R, de Boer HC, Segers D, Huisman M, et al. Shear stress-induced changes in atherosclerotic plaque composition are modulated by chemokines. J Clin Invest. 2007;117:616-26.

22. Fuster V, Moreno PR, Fayad ZA, Corti R, Badimon JJ. Atherothrombosis and high-risk plaque: part I: evolving concepts. J Am Coll Cardiol. 2005;46:937-54.

23. Sucosky P, Balachandran K, Elhammali A, Jo H, Yoganathan AP. Altered shear stress stimulates upregulation of endothelial VCAM-1 and ICAM-1 in a BMP-4 and TGF-beta1-dependent pathway. Arterioscler Thromb Vasc Biol. 2009;29: 254-60.

24. Yoshioka M, Yuasa S, Matsumura K, Kimura K, Shiomi T, Kimura N, et al Chondromodulin-I maintains cardiac valvular function by preventing angiogenesis. Nat Med. 2006;12:1151-9.

25. Aikawa E, Nahrendorf M, Figueiredo JL, Swirski FK, Shtatland T, Kohler RH, et al. Osteogenesis associates with inflammation in early-stage atherosclerosis evaluated by molecular imaging in vivo. Circulation. 2007;116:2841-50 